Geometry \& Topology

Volume 2 (1998) 65-77

Published: 11 May 1998

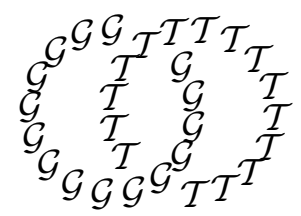

\title{
Group negative curvature for 3-manifolds with genuine laminations
}

\author{
DAVID GABAI \\ WILliam H. KAZEZ \\ California Institute of Technology \\ Pasadena, CA 91125-0001 USA \\ and \\ University of Georgia \\ Athens, GA 30602, USA
}

Email: gabai@cco.caltech.edu and will@math.uga.edu

\begin{abstract}
We show that if a closed atoroidal 3-manifold $M$ contains a genuine lamination, then it is group negatively curved in the sense of Gromov. Specifically, we exploit the structure of the non-product complementary regions of the genuine lamination and then apply the first author's Ubiquity Theorem to show that $M$ satisfies a linear isoperimetric inequality.
\end{abstract}

AMS Classification numbers Primary: 57M50

Secondary: 57R30, 57M07, 20F34, 20F32, 57M30

Keywords: Lamination, essential lamination, genuine lamination, group negatively curved, word hyperbolic

Proposed: Jean-Pierre Otal

Seconded: Robion Kirby, Michael Freedman
Received: 5 August 1997

Revised: 9 May 1998

Copyright Geometry and Topology 


\section{$0 \quad$ Introduction}

In 1985 the notion of manifold with essential lamination was introduced [11] to simultaneously generalize the notion of Haken manifold or manifold with Reebless foliation. A longstanding goal has been to generalize to laminar manifolds various results established for Haken manifolds. See [11]; [4], [2]; [3], [7]; for some results in this direction. In particular, a major goal is to show that atoroidal laminar manifolds have metrics of constant negative curvature, thereby generalizing Thurston's hyperbolization theorem [18]. Our main result shows that at the level of the fundamental group, an atoroidal 3-manifold is negatively curved (ie Gromov hyperbolic) provided that it has a genuine essential lamination, ie a lamination which is not a Reebless foliation split open along leaves.

Theorem 0.1 If $M$ is a closed atoroidal genuinely laminar 3-manifold, then $\pi_{1}(M)$ is word hyperbolic.

Remarks 0.2 i) Recall that $M$ is atoroidal if it contains no immersed $\pi_{1}-$ injective torus, equivalently $\pi_{1}(M)$ contains no rank-2 free abelian subgroup.

ii) Manifolds with essential laminations are far more plentiful then manifolds with incompressible surfaces, eg see [17], [5], [20], [11] and section 1 of the survey [6]. Gabai and Mosher [16] showed that if $k$ is a hyperbolic knot in $S^{3}$, then off of a line in Dehn surgery space, all manifolds obtained by surgery on $k$ have an essential lamination. As of this writing there is no known example of an irreducible, atoroidal, 3-manifold with infinite $\pi_{1}$ which does not have an essential lamination. Although there do exist explicit examples not known to have essential laminations. See section 1 of [6].

iii) A genuine lamination [9] is an essential lamination $\lambda$ which cannot be trivially extended to a foliation. This means that some closed complementary region of $M-\lambda$ is not an $I$-bundle. Manifolds with genuine laminations include manifolds with pseudo-Anosov flows, and by Gabai and Mosher [16] for each hyperbolic knot $k \in S^{3}$ off at most two lines in its Dehn surgery space, all manifolds obtained by surgery.

Haken manifolds are compact orientable irreducible 3-manifolds that contain an embedded incompressible surface. Such a surface $S$ is always an essential lamination and is a genuine lamination except in the case that its closed complementary region is an $I$-bundle. In this case $S$ lifts to a fibre of a fibration in a 2 -fold covering of the manifold. If $\mathrm{M}$ is atoroidal, then by [19], this covering has a pseudo-Anosov flow and hence a genuine lamination. Thus a 
closed atoroidal Haken manifold is 2-fold covered by a manifold with a genuine essential lamination.

iv) It is the non $I$-bundle complementary region that allows one to get a grip on the manifold and thereby extend to manifolds with genuine laminations various known properties of Haken manifolds, see [9], [10]. We remarked in [9] that the non $I$-bundle part makes the manifold "reek of negative curvature". The goal of this paper is to make that remark more precise.

v) Thurston's hyperbolization theorem asserts that atoroidal Haken manifolds have hyperbolic structures. Subsequently Bestvina, Feighn [1] gave an elementary argument establishing group negative curvature for such manifolds.

Idea of the Proof of Theorem 1 Suppose that the genuine lamination $\lambda$ is obtained by splitting open a singular foliation $\mu$ along the singular leaves where each singular leaf is a trigon $\times S^{1}$. Let $C$ be the singular locus of $\mu$. The Ubiquity Theorem of [8] asserts that there exists constants $K, L$ such that if $D$ is a least area disk with length $(\partial D) / \operatorname{area}(D)<L$, then $|E \cap C| / \operatorname{area}(D)>$ $K$ where $E$ is any disk which spans $\partial D$. For reasonable disks, this means that after homotopy of $D$ rel $\partial D$, the induced singular foliation on $D$ has at least $K$ area $(D) 3$-prong complementary regions and $\mu \mid D$ has no circle leaves. The Poincaré-Hopf index formula then implies that $\partial D$ is tangent to $\mu$ in at least $K \operatorname{area}(D) / 3$ different spots. This in turn implies that length $(\partial D)>$ $K^{\prime} K$ area $(D) / 3$ and hence length $(\partial D) / \operatorname{area}(D)>K^{\prime} K / 3$, for some constant $K^{\prime}$ which is defined independently of reasonable $D$. For example, $K^{\prime}$ can be taken to be the minimal distance between tangencies along $\partial D$. In summary, area $(D)$ is approximated by the geometric intersection number of $\partial D$ and $C$. The latter is approximately the number of tangencies of $\partial D$ with $\mu$. This in turn gives a lower bound for length $(\partial D)$. Therefore, $M$ satisfies a linear isoperimetric inequality, and hence $\pi_{1}(M)$ is negatively curved by Gromov [12].

The actual proof is not much different. Since $\lambda$ is genuine, there exists a finite, non-empty collection of characteristic annuli embedded in $M-\lambda$, which separates off the $I$-bundle part of the complement of $\lambda$ from the non $I$-bundle part (Lemma 1.3). Taking $C$ to be the union of cores of these annuli, we obtain length and area approximations in section 2 similar to those of the previous paragraph to conclude that $M$ satisfies a linear isoperimetric inequality.

The authors would like to dedicate this paper to David Epstein on the occasion of his 60th birthday.

The first author was partially supported by NSF Grant DMS-9505253 and the MSRI.

Geometry and Topology, Volume 2 (1998) 


\section{$1 \quad$ Preliminaries}

Notation 1.1 Let $\stackrel{\circ}{E}$ denote the interior of $E$ and $|X|$ denote the number of connected components of $X$, if $X$ is a space, or the number of elements of $X$ if it is just a set. Let $\bar{B}$ denote the closure of $B$, and $I$ denote $[0,1]$.

In this section we remind the reader of several fundamental properties of essential laminations.

Definition 1.2 A 2-dimensional lamination $\lambda$ in a $3-$ manifold $M$ is a foliation of a closed subset of $M$. More precisely $M$ has charts of the form $\mathbb{R}^{2} \times \mathbb{R}$, such that $\lambda \mid \mathbb{R}^{2} \times \mathbb{R}$ is the product lamination $\mathbb{R}^{2} \times T$, where $T$ is a closed subset of $\mathbb{R}$. A component of $M-\lambda$ is called a complementary region. A closed complementary region is a component $V$ of $M-\lambda$ metrically completed with respect to the induced path metric. Topologically it is $V$ together with its boundary leaves. We will assume that the leaves of $\lambda$ are smoothly immersed in $M$, although the transverse structure may only be $C^{0}$.

A lamination $\lambda$ in the closed orientable 3-manifold $M$ is essential [11] if there are no 2 -sphere leaves, each leaf is $\pi_{1}$-injective, $M-\lambda$ is incompressible and each closed complementary region is end-incompressible. The closed complementary region $V$ is end-incompressible if for every proper map $f: D^{2}-x \rightarrow V$, $x \in \partial D^{2}$ such that $f\left(\partial D^{2}-x\right) \subset L, L$ a leaf of $\lambda$, then there exists a proper map $g: D^{2}-x \rightarrow L$ such that $g\left|\partial D^{2}-x=f\right| \partial D^{2}-x$.

Let $X$ be a codimension- 0 submanifold of a $3-$ manifold $V$. The horizontal boundary of $X$ is defined to be $X \cap \partial V$ and is denoted $\partial_{h} X$. The vertical boundary of $X$ is defined to be the closure in $\partial X$ of $\partial X-\partial_{h} X$ and is denoted $\partial_{v} X$. Typically $V$ will be a closed complementary region so that $\partial_{h} X=X \cap \lambda$ and $\partial_{v} X$ will be a union of annuli. The pair $\left(X, \partial_{h} X\right)$ is called an $I$-bundle if $X$ is the total space of an $I$-bundle over a surface $S$ in such a way that $\partial_{h} X$ consists of the boundary points of the $I$-bundle fibres.

A product disk is defined to be a proper embedding of $(I \times I, \partial I \times I, I \times \partial I)$ into $\left(X, \partial_{v} X, \partial_{h} X\right)$ such that each component of $\partial I \times I$ is mapped to an essential arc in $\partial_{v} X$. A product disk is essential if it is not parallel, keeping $\partial I \times I$ in $\partial_{v} X$ and $I \times \partial I$ in $\partial_{h} X$, to a disk in $\partial_{v} X$. 
Lemma 1.3 Let $V$ be the disjoint union of the closed complementary regions of an essential lamination $\lambda$. There exists a unique (up to isotopy in $V$ ) finite collection $\mathcal{A}=A_{1} \cup \cdots \cup A_{n}$ of properly embedded annuli in $V$ such that

(1) $V=\mathcal{G} \cup \mathcal{I}$ where $\mathcal{G} \cap \mathcal{I}=\partial_{v} \mathcal{G}=\partial_{v} \mathcal{I}=\mathcal{A}$.

(2) $\left(\mathcal{I}, \partial_{h} \mathcal{I}\right)$ is an $I$-bundle over a possibly noncompact or disconnected surface. No component of $\left(\mathcal{I}, \partial_{h} \mathcal{I}\right)$ is an $I$-bundle over a compact surface with non-empty boundary.

(3) $\left(\mathcal{G}, \partial_{h} \mathcal{G}\right)$ is compact, has no components homeomorphic to $\left(D^{2} \times I, D^{2} \times\right.$ $\partial I)$ and contains no essential product disks.

Proof This follows almost immediately from [14] or the Generalized Splitting Theorem of [15]. We shall only point out the minor differences.

Their theorem is stated for compact pairs $\left(V, \partial_{h} V\right)$. If $N(B)$ is a fibred neighborhood of a branched surface which carries $\lambda$, then $N(B) \cap V$ gives an $I-$ bundle fibring of the ends of $\left(V, \partial_{h} V\right)$. Thus their result may be applied to the finitely many components of the space $\left(V, \partial_{h} V\right)$ split along $\partial_{v}(N(B) \cap V)$ which are compact.

Let $\left(\mathcal{I}, \partial_{h} \mathcal{I}\right)$ be those finitely many components of the maximal $I$-bundle of $\left(V, \partial_{h} V\right)$ given by [15], [14] which either contain an end of $V$ or are of the form $S \times I$ where $S$ is a closed surface. Let $\mathcal{G}=V-\left(\mathcal{I}-\partial_{v} \mathcal{I}\right)$ and $\partial_{h} \mathcal{G}=\mathcal{G} \cap \partial_{h} V$. (3) follows by the maximality of $\mathcal{I}$. By construction $\mathcal{I}$ has no $D^{2} \times S^{1}$ components and consequently establishing uniqueness is routine.

Figures 1.1 and 1.2 show examples of the decomposition $V=\mathcal{G} \cup \mathcal{I}$. Notice in Figure 1.1 that compact $I$-bundles are part of the guts $\mathcal{G}$. In Figure $1.2 \mathcal{I}$ is maximal among $I$-bundles with no compact components, but there are two different ways to add compact $I$-bundles to $\mathcal{I}$.

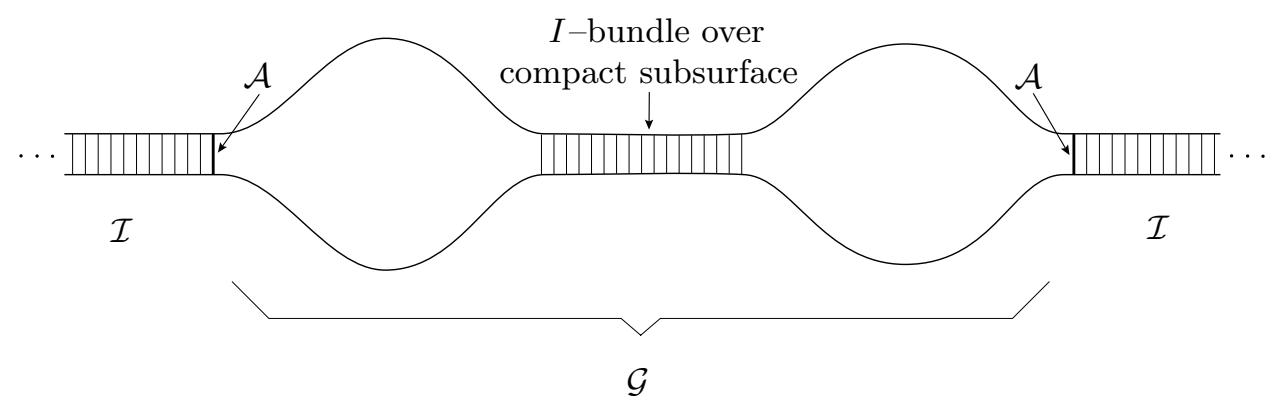

Figure 1.1 

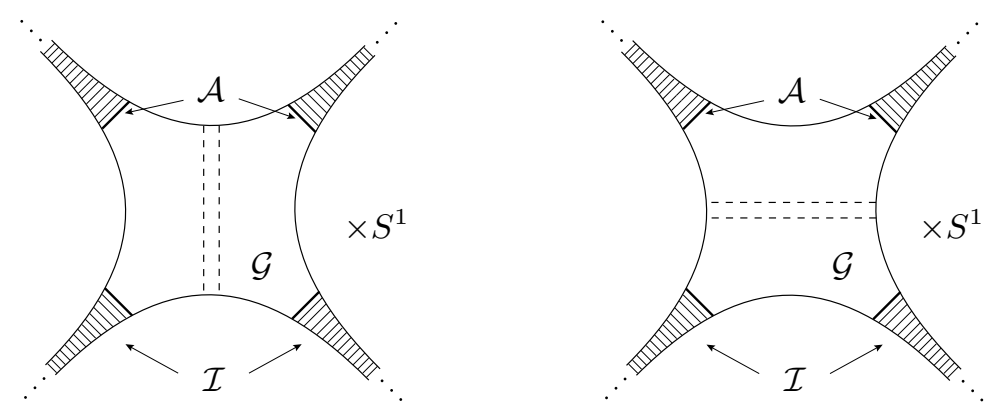

Figure 1.2

Definition 1.4 Let $\mathcal{C}$ denote the union of the cores of the $A_{i}$ 's. $\mathcal{I}$ together with its $I$-bundle structure is called the interstitial bundle of $\lambda . \mathcal{G}$ is called the guts of $\lambda$.

Definition 1.5 A genuine lamination is an essential lamination in $M$ with non-empty guts. In other words, a genuine lamination is an essential lamination which is not just a Reebless foliation with some leaves split open.

Lemma 1.6 If $M$ has an essential lamination $\lambda_{1}$, then $M$ has an essential lamination $\lambda$ which is nowhere dense and has no isolated leaves. If $\lambda_{1}$ is genuine, then $\lambda$ can be taken to be genuine.

Proof If $M-\lambda_{1}=\emptyset$, then by first Denjoy splitting open a leaf we can assume that $\lambda_{1}$ is not all of $M$. Let $\lambda_{2}$ be a sublamination of $\lambda_{1}$ such that each leaf is dense in $\lambda_{2}$. If $\lambda_{2}$ is a single compact leaf, then we may assume that it is 2 -sided by passing, if necessary, to a double cover. Finally replace it by $\lambda=\lambda_{2} \times T$, where $T$ is a Cantor set. Use the fact that a sublamination of of an essential lamination is essential by [11] to show that $\lambda$ is essential. Finally if $\lambda_{1}$ is genuine, then so is $\lambda$.

Remark 1.7 i) The feature of no isolated leaves allows us to assume that distinct $A_{i}$ 's do not intersect.

ii) Let $\alpha$ be a properly embedded arc in a closed complementary region $V$ of $\lambda$. We say that $\alpha$ is efficient if $\alpha$ cannot be homotoped rel $\partial \alpha$ into $\partial V$, via a homotopy supported in $V$.

To establish Theorem 0.1 we need the following special case of Theorem 1 of [11]. 
Theorem 1.8 Let $\lambda$ be an essential lamination in $M$, then

i) Interstitial fibres are efficient arcs.

ii) If $I$ is an efficient arc, then $I$ cannot be homotoped (in $M$ ) rel $\partial I$ to lie in a leaf.

iii) If $C_{i}$ is a core of $A_{i}$, then $0 \neq\left[C_{i}\right] \in \pi_{1}(M)$.

iv) If $\psi$ is a closed efficient transversal curve (ie $\psi$ is transverse to $\lambda$ and the closure of each component of $\psi-\lambda$ is an efficient arc), then $0 \neq[\psi] \in \pi_{1}(M)$.

\section{$2 \quad$ Proof of Theorem 0.1}

Let $\lambda$ be a genuine lamination in the closed 3-manifold $M$. By Lemma 1.6 we can assume that $\lambda$ is nowhere dense and that no leaf of $\lambda$ is isolated. As in section 1 we let $\left\{A_{i}\right\}$ be the finite set of characteristic annuli, $\mathcal{A}=\cup A_{i}$ and let $\mathcal{C}$ be the union of the cores of the $A_{i}$ 's.

Fix a Riemannian metric on $M$. By Gromov [12], $\pi_{1}(M)$ is word hyperbolic if there exists $L>0$ such that for each least area mapping of a disk $f: D \rightarrow M$, length $(\partial f) / \operatorname{area}(f)>L$.

Lemma 2.1 Let $K>0$. There exists $\epsilon>0$ with the following property. If the set of least area functions $f: D \rightarrow M$ satisfying

(1) $d(\partial f(\partial D), \mathcal{A})>\epsilon$,

(2) length $(\partial f \mid Y)>\epsilon$ for each component $Y$ of $f^{-1}(\mathcal{G}) \cap \partial D$,

(3) $\partial f$ is transverse to $\lambda$ and

(4) $f(\partial D)$ intersects $\mathcal{I}$ in interstitial fibres

all satisfy the isoperimetric inequality length $(\partial f) / \operatorname{area}(f)>K$, then $M$ is negatively curved. 
Proof First observe that there exists $C_{1}$, and $\epsilon$ such that if $\partial g_{0}: S^{1} \rightarrow M$ is any smooth immersion, then $\partial g_{0}$ is homotopic to $\partial g_{1}$ via $G: S^{1} \times I \rightarrow M$ such that $\partial g_{1}$ satisfies the conclusions of $(1)-(4)$, area $(G) \leq C_{1} \operatorname{length}\left(\partial g_{0}\right)$ and length $\left(\partial g_{0}\right) \geq \epsilon$ length $\left(\partial g_{1}\right)$. (Hint: Think of $\lambda$ as being carried in an extremely thin fibred neighborhood of the smooth branched surface $B$, with the various components of $\mathcal{A}$ lying in tiny neighborhoods of sparsely placed embedded curves in $B$. The homotopy of $\partial g_{0}$ to $\partial g_{1}$ is more or less one which first makes $\partial g_{0}$ piecewise geodesic, then pushes $\partial g_{0}$ off a small neighborhood of $\mathcal{A}$ and is transverse to $B$.)

Let $g_{0}: D \rightarrow M$ be an arbitrary least area map. Suppose that $\partial g_{1}$ is as above, and $g_{1}: D \rightarrow M$ is any least area map which extends $\partial g_{1}$. Let $K_{1}=\min \{K, 1\}$. Then

$$
K<\operatorname{length}\left(\partial g_{1}\right) / \operatorname{area}\left(g_{1}\right) \leq \frac{1}{\epsilon} \operatorname{length}\left(\partial g_{0}\right) / \operatorname{area}\left(g_{1}\right)
$$

and hence

$$
\begin{gathered}
K_{1}<\left(\frac{1}{\epsilon} \operatorname{length}\left(\partial g_{0}\right)+C_{1} \operatorname{length}\left(\partial g_{0}\right)\right) /\left(\operatorname{area}\left(g_{1}\right)+C_{1} \operatorname{length}\left(\partial g_{0}\right)\right) \\
\leq\left(\frac{1}{\epsilon}+C_{1}\right) \operatorname{length}\left(\partial g_{0}\right) / \operatorname{area}\left(g_{0}\right)
\end{gathered}
$$

which implies that length $\left(\partial g_{0}\right) / \operatorname{area}\left(g_{0}\right) \geq K_{1} /\left(\frac{1}{\epsilon}+C_{1}\right)$.

Let $E$ be any smooth branched immersed disk that spans $\partial D$ and minimizes geometric intersection number with $\mathcal{C}$. In what follows (Claim 3) we establish a relationship between $|E \cap \mathcal{C}|,|E \cap \mathcal{G}|$ and length $(\partial D)$.

After a homotopy supported away from $\partial D$ we can assume that $E$ is transverse to $\lambda \cup \mathcal{A}$. Since leaves of essential laminations are $\pi_{1}$-injective and closed transverse efficient curves are homotopically nontrivial, the induced lamination $\lambda \mid E$ is a lamination by circles and properly embedded arcs. Furthermore each circle is homotopically trivial in its leaf. Therefore the standard Reeb stability argument implies the following lemma.

Packet Lemma 2.2 $\lambda \mid E$ is a union of product laminations of the form $I \times T$ or $S^{1} \times T$ where $T$ is a Cantor set in $I$ and $I \times I$ is an embedded square in $E$ with $\partial E \cap I \times I=\partial I \times I$ or $S^{1} \times I$ is an embedded annulus in $\stackrel{\circ}{E}$. Furthermore the various $I \times I$ 's and the $S^{1} \times I$ 's are pairwise disjoint.

Let $\mathcal{G}, \mathcal{I}, \mathcal{A}$, and $\mathcal{C}$ be as in Lemma 1.3. The proof of Theorem 0.1 consists of an analysis of the pullback of these sets to $E$. These pullbacks are denoted $G, J, A$, and $C$ respectively. Claims 0,1 , and 2 show how to homotop $E$ so that it efficiently intersects $\mathcal{G}, \mathcal{I}, \mathcal{A}$, and $\mathcal{C}$. 
Claim $0 \quad E$ can be homotoped rel $\partial E$ to eliminate circle components of $\lambda \cap E$.

Proof of Claim 0 Let $E_{1}, \cdots, E_{n}$ be a finite disjoint union of embedded disks in $E$, which contain all the circle components of $\lambda \mid E$ and are disjoint from the non-circle leaves. The $\pi_{1}$-injectivity of leaves of $\lambda$, implies that $E$ can be homotoped rel $E-\cup E_{i}$ to eliminate the circle leaves, ie after homotopy $E \cap \lambda=\left(E-\cup E_{i}\right) \cap \lambda$. Notice that such a homotopy does not increase the number of arc components of $E \cap \mathcal{A}$. The resulting mapping of $E$ will be a branched immersion transverse to $\lambda \cup \mathcal{A}$.

Claim $1 E$ can be homotoped rel $\partial E$ so that if $B$ is a component of $E \cap \mathcal{A}$, then $B$ is an arc which connects distinct leaves of $\lambda \mid E$. Furthermore $|B \cap C| \leq 1$ and $\lambda \mid E$ still has no circle components.

Proof of Claim 1 If $B$ is an innermost circle component of $E \cap \mathcal{A}$ let $E_{1} \subset \stackrel{\circ}{E}$ be an embedded disk such that $B \subset \stackrel{\circ}{E_{1}}$ and $E_{1} \cap(\lambda \cup \mathcal{A})=B$. The $\pi_{1}-$ injectivity of $\mathcal{A}$ implies that a homotopy of $E$ supported in $E_{1}$ eliminates $B$ and the resulting $E_{1}$ will be disjoint from $\lambda \cup A$.

Now suppose that $B$ is an arc which connects the same leaves of $\lambda \mid E$. By passing to possibly another such one we can find an embedded disk $E_{1} \subset E$ with $\partial E_{1}$ the union of $B$ and an arc in a leaf of $\lambda \cap E$. By Theorem $1.8 B$ is a homotopically inessential arc in $A$. Since no leaf of $\lambda$ is isolated there is small neighborhood $N\left(E_{1}\right)$ of $E_{1}$ such that $N\left(E_{1}\right) \cap A=B$. Thus a homotopy of $E$ supported in $N\left(E_{1}\right)$ eliminates this component $B$ of $E \cap A$ without introducing other components of $E \cap A$. Furthermore, the homotopy can be carried out so that $\lambda \mid E$ continues to have no circle components. Therefore we can assume that if $B$ is a component of $E \cap \mathcal{A}$, then $B$ is an arc which connects distinct leaves of $\lambda \mid E$. Since $|E \cap C|$ is minimal it follows that $|B \cap C| \leq 1$.

Claim 2 After a homotopy of $E$ relative to $\partial E$, there is no component $G_{1}$ of $G$ that is contained in $\stackrel{\circ}{E}$ and is a disk homeomorphic to $I \times I$ in such a way that $G_{1} \cap \partial_{v} G=\partial I \times I$, where $0 \times I$ is mapped to an essential arc in $\mathcal{A}$.

Proof of Claim 2 Suppose such a $G_{1}$ exists. Let $K_{i}=i \times I$. By Theorem 1.8 $K_{0}$ cannot be homotoped rel $\partial K_{0}$ into a leaf of $\lambda$. It follows that $K_{1}$ is also essential. An application of the Loop Theorem shows that either $\mathcal{G}$ contains an essential product disk or there exists a relative homotopy in $\mathcal{G}$ deforming $G_{1}$ into $\mathcal{A}$. (Ie a homotopy $F:(I \times I) \times I \rightarrow \mathcal{G}$ such that $F_{0}=f\left|G_{1}, F_{t}\right| i \times I=$ $f \mid i \times I$ for $i \in\{0,1\}, F_{t}\left|I \times i \subset \partial_{h}\right| \mathcal{G}$ for $i \in\{0,1\}$ and $F_{1} \mid I \times I \subset \mathcal{A}$.) By Lemma 1.3 (3), the latter must occur. Therefore after a homotopy of $E$ relative to $\partial E$, the number $|E \cap C|$ gets reduced by 2 contradicting the minimality hypothesis. 
Figure 2.1 shows the $G_{i}$ 's, in grey, and the $J_{j}$ 's, in white, as subsets $E$. Points of $G \cap J$ which map to $\mathcal{C}$ are indicated with dots. By Claim 2, regions like $G_{1}$ can be removed by an isotopy, and regions like $G_{2}$ can not exist. Note that components of $J \mid E$ need not be $I$-bundles. Indeed had $\lambda$ been a singular foliation $\mu$ split open, then non $I$-bundle components of $J \mid E$ could have arisen from tangencies of $D$ with $\mu$.

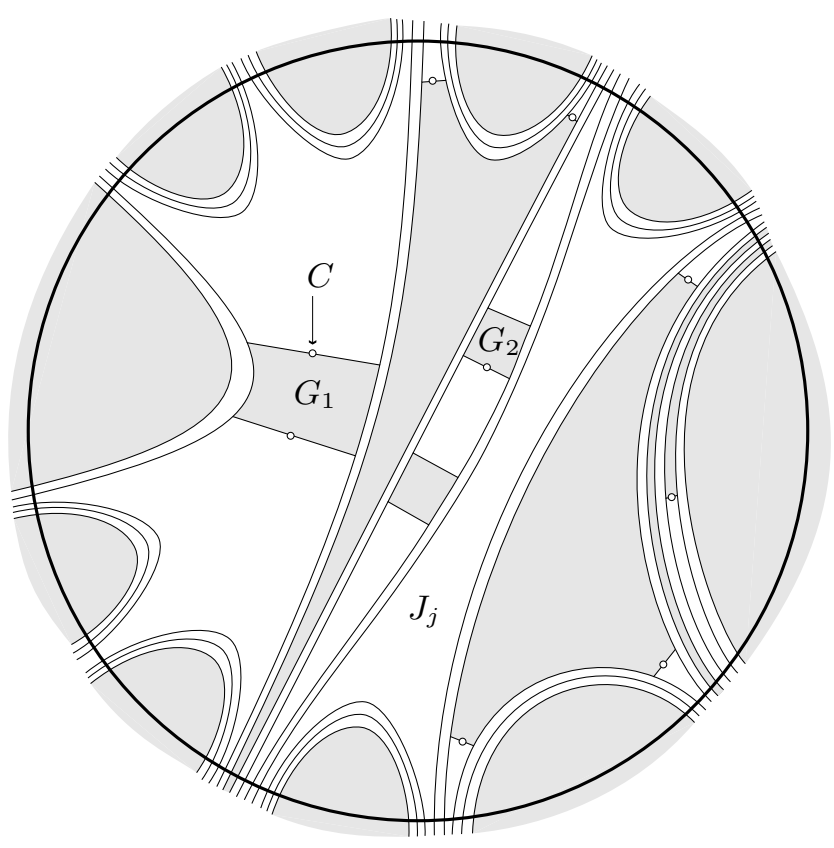

Figure 2.1

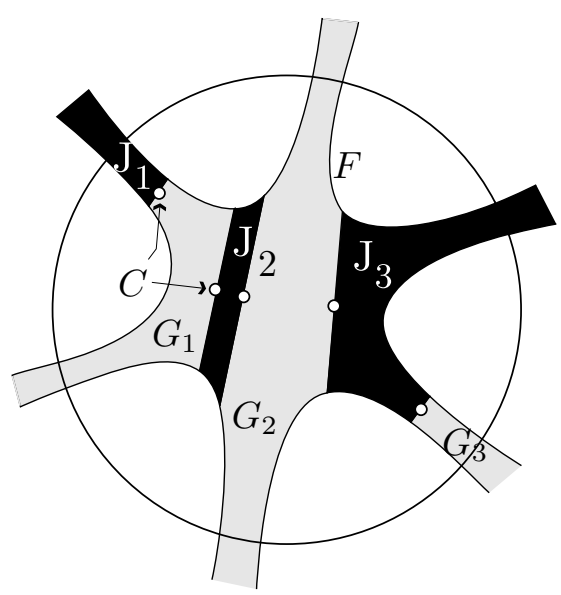

Figure 2.2 
If $F$ is a component of $E-\lambda$, define the index $I(F)$ to be $1-\frac{1}{2}|F \cap \partial E|$. There are only finitely many complementary regions of non zero index by the Packet Lemma and Claim 1. Since $E$ is a disk, $\sum_{F} I(F)=1$. The regions of index $1 / 2$ are in 1-1 correspondence with the outermost arcs of $\lambda \cap E$, moreover these regions are components of $G$. Let $N$ be the number of such regions. Since $1=\sum_{F} I(F)=\sum_{I(F) \leq 0} I(F)+\sum_{I(F)>0} I(F)$ it follows that $N=2-2 \sum_{I(F) \leq 0} I(F)$.

Claim $3 \quad N>\frac{1}{3}|E \cap C|-\frac{1}{3 \epsilon} \operatorname{length}(\partial E)$.

Proof of Claim 3 Let $F$ be a closed complementary region of $E-\lambda$ and let $G_{1}, \ldots, G_{p}$ (respectively $J_{1}, \ldots, J_{q}$ ) be the components of $G \cap F$ (respectively $J \cap F)$. See Figure 2.2.

Define $I\left(G_{i}\right)=1-\frac{1}{2}\left|G_{i} \cap(A \cup \partial E)\right|$ and $I\left(J_{i}\right)=1-\frac{1}{2}\left|J_{i} \cap(A \cup \partial E)\right|$, thus $I(F)=$ $\sum_{i} I\left(G_{i}\right)+\sum_{j} I\left(J_{j}\right)$. By construction $\partial E$ intersects $J$ only in interstitial fibres and by Theorem 1.8 such arcs cannot be homotoped, fixing endpoints, into $\lambda$, thus

(1) $I\left(J_{j}\right) \leq 0$ for all $\mathrm{j}$.

(2) If $I\left(G_{i}\right)<0$ then $-I\left(G_{i}\right) \geq \frac{1}{6}\left|G_{i} \cap C\right|$.

(3) If $I\left(G_{i}\right)=0$ then $\left|G_{i} \cap C\right| \leq\left|G_{i} \cap \partial E\right|$.

(4) If $I\left(G_{i}\right)>0$ then $G_{i}=F, I\left(G_{i}\right)=I(F)=\frac{1}{2}$ and $\left|G_{i} \cap C\right|=0$.

(In the following sums we suppress double subscript notation by using the same notation $\bigcup_{i} G_{i} \cup \bigcup_{j} J_{j}$ for the various different regions $F$.) We have,

$$
\begin{gathered}
N=2-2 \sum_{I(F) \leq 0} I(F) \\
>2 \sum_{I(F) \leq 0}\left(\sum_{i}\left(-I\left(G_{i}\right)\right)+\sum_{j}\left(-I\left(J_{j}\right)\right)\right) \\
\geq 2 \sum_{I(F) \leq 0} \sum_{i}\left(-I\left(G_{i}\right)\right) \\
=2 \sum_{I(F) \leq 0} \sum_{I\left(G_{i}\right)<0}\left(-I\left(G_{i}\right)\right) \\
\geq 2 \sum_{I(F) \leq 0} \sum_{I\left(G_{i}\right)<0} \frac{1}{6}\left|G_{i} \cap C\right|
\end{gathered}
$$




$$
\begin{gathered}
=\frac{1}{3} \sum_{I(F) \leq 0}\left(\sum_{I\left(G_{i}\right) \leq 0}\left|G_{i} \cap C\right|-\sum_{I\left(G_{i}\right)=0}\left|G_{i} \cap C\right|\right) \\
=\frac{1}{3}|E \cap C|-\frac{1}{3} \sum_{I(F) \leq 0} \sum_{I\left(G_{i}\right)=0}\left|G_{i} \cap C\right| \\
\geq \frac{1}{3}|E \cap C|-\frac{1}{3} \sum_{I(F) \leq 0} \sum_{I\left(G_{i}\right)=0}\left|G_{i} \cap \partial E\right| \\
\geq \frac{1}{3}|E \cap C|-\frac{1}{3} \sum_{F} \sum_{G_{i}}\left|G_{i} \cap \partial E\right| \\
=\frac{1}{3}|E \cap C|-\frac{1}{3}|G \cap \partial E| \quad \text { (by Le } \\
\geq \frac{1}{3}|E \cap C|-\frac{1}{3 \epsilon} \operatorname{length}(\partial E) .
\end{gathered}
$$

(by Lemma 2.1)

\section{Proof of Theorem 0.1}

By the Ubiquity Theorem, [8], there exists $K, L>0$ such that if $D$ is a least area disk such that length $(\partial D) / \operatorname{area}(D)<L$, then

$$
|E \cap \mathcal{C}| / \operatorname{area}(D)>K
$$

where $E$ is any disk which spans $\partial D$. This means that for disks of small isoperimetric ratio, up to multiplicative constants, the wrapping number of $\partial D$ with $\mathcal{C}$ is more or less the same as area $(D)$.

Since there are $N$ regions of $E$ with index $\frac{1}{2}, \partial E \cap G$ consists of at least $N$ components and therefore,

$$
\begin{array}{rr}
\operatorname{length}(\partial D)=\text { length }(\partial E) \geq \epsilon N & \text { (by Lemma 2.1) } \\
\geq \frac{\epsilon}{3}|E \cap C|-\frac{1}{3} \operatorname{length}(\partial E) & \text { (by Claim 3) } \\
\geq \frac{K \epsilon}{3} \operatorname{area}(D)-\frac{1}{3} \operatorname{length}(\partial D) . & \text { (by } \left.\left(^{*}\right)\right)
\end{array}
$$

It follows that

$$
\operatorname{length}(\partial D) \geq \frac{K \epsilon}{12} \operatorname{area}(D)
$$




\section{References}

[1] M Bestvina, M Feighn, A Combination Theorem for Negatively Curved Groups, J. Diff. Geom. 35 (1992) 85-101

[2] M Brittenham, Essential Laminations in Seifert Fibred Spaces, Topology 32 (1993) 61-85

[3] M Brittenham, Essential Laminations and Haken Normal Form I, Pacific J. 168 (1995) 217-234

[4] E Claus, Thesis, U Texas, Austin (1990)

[5] C Delman, R Roberts, (in preparation)

[6] D Gabai, Problems in the Geometric Theory of Foliations and Laminations on 3-Manifolds, from: "Geometric Topology", Vol 2 (W H Kazez Ed.) AMS/IP (1997) 1-33

[7] D Gabai, Essential Laminations and Kneser Normal Form, (in preparation)

[8] D Gabai, The Ubiquitous Nature of Quasi-Minimal Semi-Euclidean Laminations in 3-Manifolds, (to appear) Surveys in Differential Geometry, 5 (International Press)

[9] D Gabai, W H Kazez, Homotopy, Isotopy and Genuine Laminations of 3Manifolds, from: "Geometric Topology", Vol 1 (W H Kazez Ed.) AMS/IP (1997) 123-138

[10] D Gabai, W H Kazez, Finiteness of the Mapping Class Group for Atoroidal 3-Manifolds with Genuine Laminations, (preprint)

[11] D Gabai, U Oertel, Essential Laminations in 3-Manifolds, Ann. Math. 130 (1989) 41-73

[12] M Gromov, Hyperbolic Groups, MSRI Pubs. 8 75-2-64

[13] W Haken, Theorie der Normal Flachen, Acta. Math. 105 (1961) 245-375

[14] K Johannson, Homotopy Equivalences of 3-Manifolds with Boundary, Springer LNM 761 (1979)

[15] W Jaco, P Shalen, Seifert Fibered Spaces in 3-Manifolds, Mem. AMS 21 (1979)

[16] L Mosher, Combintorics of Pseudo-Anosov Flows, (in preparation)

[17] R Naimi, Constructing essential laminations in 2-bridge knot surgered 3manifolds, Pacific J. Math. 180 (1997) 153-186

[18] W P Thurston, Three-Dimensional Manifolds, Kleinian Groups and Hyperbolic Geometry, Proc. Symp. Pure Math. 39 (1979) 87-111

[19] W P Thurston, On the Geometry and Dynamics of Diffeomorphisms of Surfaces , Bull. Amer. Math.Soc. 19 (1988) 417-431

[20] Y Q Wu, Dehn Surgery on Arborescent Knots, J. Diff. Geom. 43 (1996) 171197 